\title{
Construcción de una curva de demanda de cloro para la planta potabilizadora Jaime Díaz Quintero en La Chorrera, Panamá
}

\author{
Melgar, Yarelis \\ Universidad del Istmo \\ Ciudad de La Chorrera, Panamá \\ yymf04@gmail.com \\ Duarte, Bolívar \\ Instituto de Acueductos y Alcantarillados Nacionales \\ Ciudad de La Chorrera, Panamá \\ bolivarjduarte@gmail.com \\ López, Eduardo \\ Instituto de Acueductos y Alcantarillados Nacionales \\ Ciudad de Panamá, Panamá \\ darturo88@gmail.com \\ Gonzalo, Pulido \\ Universidad del Istmo \\ Ciudad de La Chorrera, Panamá \\ gpulido@udelistmo.edu
}

\begin{abstract}
This work presents the experimental obtaining of the chlorine demand curve of the raw water flowing into the IDAAN Jaime Díaz Quintero drinking water treatment plant during the rainy season, in order to understand and apply the experimental procedures involved, interpret the observed behavior of chlorine demand in raw water and associate it to the chemical species present in the raw water. The free residual chlorine present in twelve raw water samples with different concentrations of calcium hypochlorite stock solution at $65 \%$ purity was evaluated using the DPD-colorimeter method. The break point of each curve was obtained by simple linear regression of the data obtained. The results show a break point of $3 \mathrm{mg} / \mathrm{L}$ of added chlorine dose. From the break point, the chlorine demand curve has an upward behavior, which is possibly attributed to the presence of low concentrations of organic nitrogen detected in the history of the last six months of nitrate and nitrite data.
\end{abstract}


Through this study, it is expected to find the average chlorine demand curve and obtain an indicator of the organic load of the raw water, since the greater the displacement of the breakpoint, the greater the organic load.

Keywords: Chlorine demand curve, break point, chlorine demand.

\section{Resumen}

Este trabajo presenta la obtención experimental de la curva de demanda de cloro del agua cruda afluente a la potabilizadora IDAAN Jaime Díaz Quintero en temporada lluviosa, a fin de entender y aplicar los procedimientos experimentales involucrados, interpretar el comportamiento observado de demanda de cloro en agua cruda y asociarlo a las especies químicas presentes en el agua cruda. Se evaluó el cloro residual libre, presente en doce muestras de agua cruda con diferentes concentraciones de solución madre de hipoclorito de calcio al $65 \%$ de pureza, mediante el método DPD-colorímetro. El punto de quiebre de cada curva se obtuvo mediante regresión lineal simple de los datos obtenidos. Los resultados muestran un punto de quiebre de $3 \mathrm{mg} / \mathrm{L}$ de dosis de cloro añadido. Desde el punto de quiebre, la curva de demanda de cloro tiene un comportamiento ascendente, que se atribuye posiblemente a la presencia en bajas concentraciones de nitrógeno orgánico detectadas en el historial de los últimos seis meses de datos de nitratos y nitritos. A través de este estudio se espera encontrar la curva de demanda de cloro promedio y obtener un indicador de la carga orgánica del agua cruda, pues a mayor desplazamiento del punto de quiebre, mayor carga orgánica.

Palabras claves: Curva de demanda de cloro, punto de quiebre, demanda de cloro.

\section{INTRODUCCIÓN}

El propósito primario de la desinfección del agua es impedir la diseminación de enfermedades de origen hídrico. El cloro el desinfectante de mayor aplicación, especialmente en países en desarrollo. La cantidad de cloro que consumen las sustancias reductoras y la materia orgánica presente en el agua cruda se define como demanda de cloro Demanda de cloro = dosis de cloro - cloro residual [1], y depende especialmente la materia orgánica, que en contacto con cloro se convierte en un tema de interés en salud pública por la posible formación de trihalometanos; la dosis de cloro, tipo de residual deseado, tiempo de contacto, $\mathrm{pH}$ y temperatura. La determinación de la demanda de cloro, en el diseño 
y operación de plantas de tratamiento de agua potable, permite cuantificar la capacidad requerida de los cloradores, y sobre todo la dosis de cloro a aplicar, para obtener un residual determinado [1]-[3].

Para el análisis de cloro residual y combinado se aplica el método DPD-colorimétrico, que usa el reactivo dietil-parafenilen-diamina, que a pH 6.2-6.5 y en presencia de cloro, da una coloración roja proporcional a la concentración de cloro [4]. La medida del color se hace semi cuantitativamente por comparación con una escala de color o con un espectrofotómetro [1], [4].

En la actualidad, el Instituto de Acueductos y Alcantarillados Nacionales (IDAAN) opera 55 potabilizadoras a nivel nacional [5]; sin embargo, el uso de curvas de demanda de cloro es muy limitada. Este estudio busca que el método y resultados obtenidos en la planta potabilizadora Jaime Díaz Quintero, pueda ser replicado en otras en otras potabilizadoras del IDAAN.

\section{MÉTODO}

\section{A. Materiales}

- Reactivo DPD Cloro libre $\bullet$ Hipoclorito de calcio al 65\% de pureza 13 frascos ámbar de vidrio de $1.0 \mathrm{~L} \bullet$ Probeta de $500 \mathrm{~mL} \bullet$ Colorímetro portátil y celdas de $10 \mathrm{~mL} \bullet$ Mortero y pilón $\bullet$ Vasos químicos varios $\bullet$ Vidrio reloj $\bullet$ Matraz volumétrico $1000 \mathrm{~mL} \bullet$ Jeringas varias - Policial • Balanza analítica.

\section{B. Procedimiento general de laboratorio [6], [7]}

- Disolver $0.07692 \mathrm{~g}$ de hipoclorito de calcio al $65 \%$ de pureza en $1 \mathrm{~L}$ de agua desionizada, para preparar una solución madre de $50 \mathrm{mg} / \mathrm{L}$.

- Agregar $0.50 \mathrm{~mL}$ de agua cruda a cada frasco ámbar de $1 \mathrm{~L}$.

- Seleccionar los frascos ámbar en grupos de 2 en 2 y dispensar los $\mathrm{mL}$ de solución de para lograr la concentración de estudio, como se muestra en la tabla 1.

- Completar cada frasco en agua cruda hasta alcanzar la capacidad de $1 \mathrm{~L}$ y agitar uniformemente por espacio de 1 minuto. Repetir este procedimiento hasta completar las 12 muestras.

- Dejar reposar todas las muestras un tiempo de contacto de 30 minutos; tiempo apropiado para hacer la reacción de oxidación y destrucción del material biológico.

- Pasado el tiempo de contacto, ajustar el colorímetro portátil en cero.

- Extraer $10 \mathrm{~mL}$ de la muestra 1, añadir un sobre de reactivo DPD y agitar por 30 segundos. Pasado el tiempo, leer la concentración de cloro residual en la pantalla del equipo. Repetir para el resto de las muestras. 
- Con los valores de dosis y residual obtenidos, graficar la curva y obtener la demanda de cloro, asociada el punto de quiebre.

\section{Obtención del punto de quiebre}

El procedimiento aplicado para la obtención del punto de quiebre consiste en analizar el patrón de comportamiento de los datos obtenidos mediante regresión lineal simple, con la finalidad de encontrar el punto de intersección entre las líneas de tendencia de cada grupo de datos, el cual se interpreta como el punto de quiebre de la curva. El punto de quiebre del agua cruda de la planta, corresponde al promedio de todos los puntos de quiebres individuales de cada curva de estudio.

Tabla 1. Volumen en $\mathrm{mL}$ de solución madre para lograr la dosis de requerida por muestra

\begin{tabular}{cccccc}
\hline Muestra & Dosis de estudio $(\mathbf{m g} / \mathbf{L})$ & Solución de $(\mathbf{m L})$ & Muestra & $\begin{array}{c}\text { Dosis de estudio } \\
(\mathbf{m g} / \mathbf{L})\end{array}$ & Solución de $(\mathbf{m L})$ \\
\hline 1 & 1,00 & 20,00 & 7 & 3,00 & 60,00 \\
2 & 1,30 & 26,00 & 8 & 3,50 & 70,00 \\
3 & 1,50 & 30,00 & 9 & 4,00 & 80,00 \\
4 & 2,00 & 40,00 & 10 & 4,50 & 90,00 \\
5 & 2,30 & 46,00 & 11 & 5,00 & 100,00 \\
6 & 2,50 & 50,00 & 12 & 5,50 & 110,00 \\
\hline
\end{tabular}

\section{RESULTADOS}

Si se analizan los grupos de datos obtenidos en cada curva (figura 1), se puede observar un patrón de meseta (Figura 1.c.3), atribuible a aguas crudas con presencia de nitrógeno orgánico mayor al nitrógeno amoniacal [2]. En este comportamiento apreciable en curva 1 (Figura 1.a.) y curva 2 (Figura 1.b.), el primer grupo de datos (color verde) se caracteriza por presentar valores de residual muy próximos entre sí; mientras que, el siguiente grupo de datos (color naranja) se comporta de manera ascendente y constante. Los resultados, con $\mathrm{R}^{2}$ satisfactorios para el grupo de datos naranja y aceptables para el grupo de datos verde, sugieren un la existencia de un punto de quiebre alto [2] para una dosis de cloro añadido de $3 \mathrm{mg} / \mathrm{L}$, que se atribuye posiblemente a la presencia en bajas concentraciones de nitrógeno orgánico detectadas en el historial de los últimos seis meses de nitratos y nitritos en agua cruda para la fecha del estudio, que en promedio presentaron valores de 1.63 y $0.007 \mathrm{mg} / \mathrm{L}$, respectivamente [8].

Los valores máximos permitidos para estos parámetros, según lo establece el reglamento técnico en Panamá [9], son de $10 \mathrm{mg} / \mathrm{L} 1 \mathrm{mg} / \mathrm{L}$ de $\mathrm{N}$, respectivamente, por lo que se determinó que cumplen para la norma. La pendiente continua y ascendente a partir 
del punto de quiebre, sugiere la formación de notables cantidades de cloraminas que no desaparecen con el aumento de la dosis de cloro [2].

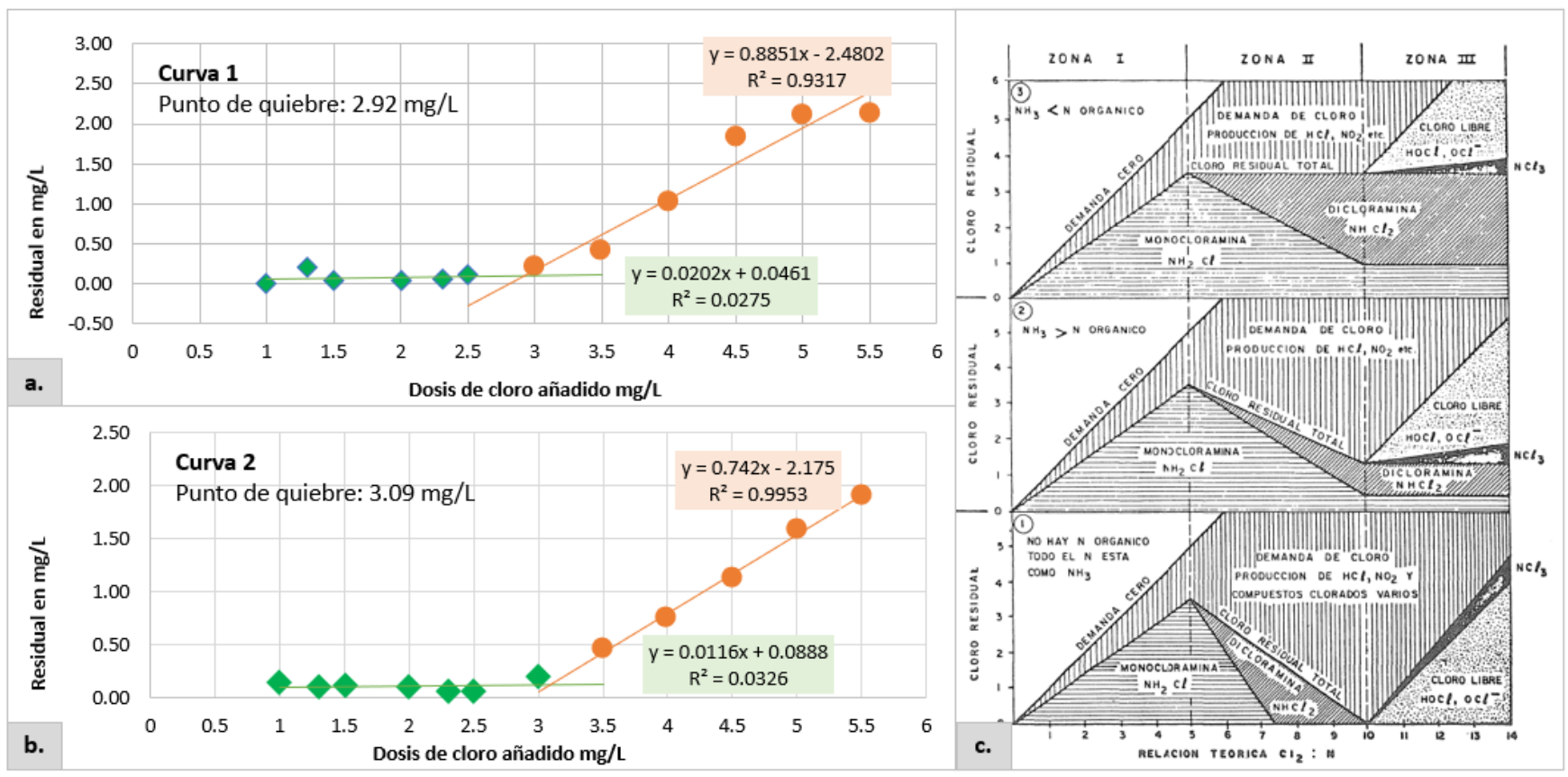

Figura 1. Resultados a. Determinación del punto de quiebre para la curva 1, b. Determinación del punto de quiebre para la curva 2, c. Curva de punto de quiebre para distintas concentraciones de nitrógeno [2]

El comportamiento de las curvas muestra además que el cloro residual libre después del punto de quiebre tiende a ser bajo. Este análisis sugiere mayor adición de dosis de cloro para completar la oxidación del sabor y olor del agua [2]; sin embargo, se debe considerar, que la operación de la potabilizadora involucra otros procesos de eliminación de materia orgánica y patógenos presentes en el agua cruda.

\section{CONCLUSIONES}

Las curvas de demanda de cloro son una herramienta eficaz en el diseño cuando el análisis se aplica al agua cruda afluente, y en la operación cuando el análisis se aplica al agua tratada efluente de las potabilizadoras. En Panamá, estas curvas deben construirse para la temporada seca y lluviosa, respectivamente. Este trabajo presenta el avance en la construcción de curvas de demanda de cloro del agua cruda afluente a la potabilizadora Jaime Díaz Quintero en temporada lluviosa. Estas curvas experimentales se construyeron a partir de los valores obtenidos de las dosis de cloro aplicadas al agua cruda versus el cloro residual libre presente en cada muestra. En cuanto al comportamiento casi continuo de las 
curvas, diferente a las típicas curvas teóricas en la literatura, se infiere que existe un punto de quiebre aparentemente alto, posiblemente influenciado por las bajas concentraciones de compuestos nitrogenados en el agua cruda analizada.

\section{Agradecimientos}

A la Secretaría Nacional de Ciencia, Tecnología e Innovación (SENACYT) por el financiamiento del proyecto "Formulación de la optimización de una planta potabilizadora mediante modelación matemática y calibración en situ", adjudicado a la Universidad del Istmo bajo el contrato IOMA 19-006, del cual se desprende esta investigación. Además, al Instituto de Acueductos y Alcantarillados Nacionales (IDAAN) por el permanente apoyo brindado en el desarrollo de la investigación.

\section{Referencias}

[1] J. A. Romero, Calidad del agua, Tercera Ed. Bogotá, Colombia: Editorial Escuela Colombiana de Ingeniería, 2009.

[2] J. Arboleda, Teoría y práctica de la purificación del agua. Bogotá, Colombia: Editorial Nomos, S. A., 1992.

[3] C. Sawyer and P. McCarty, Chemistry for environmental engineering, Third Edit. Singapore: McGrawHill, Inc., 1978.

[4] H. Luque-Torres, "Cuantificación de cloro residual en agua potable y su inhibición con tiosulfatos," Universidad Mayor de San Andrés, 2018.

[5] IDAAN, "Plataforma SIG IDAAN," 2021. https://sig-idaan.hub.arcgis.com/ (accessed May 05, 2021).

[6] American Public Health Association; American Water Works Association; Water Enrironment Federation., Standard Methods for the Examination of Water and Wastewater. Standard Methods Online, 2017.

[7] E. López, "Procedimiento general para la determinación de la demanda de cloro en la Planta Potabilizadora Jaime Díaz Quintero en La Chorrera," Panamá, República de Panamá, 2021.

[8] Departamento de Calidad de Agua de la Planta Potabilizadora Jaime Díaz Quintero (IDAAN), "Bitácora diaria de medición de parámetros," La Chorrera, Panamá, 2021.

[9] MICl, “Resolución N³5 de 6 de mayo de 2019," 2019. [Online]. Available: https://www. gacetaoficial.gob.pa/pdfTemp/28090_A/GacetaNo_28090a_20160805.pdf.

\section{Autorización y Licencia CC}

Los autores autorizan a APANAC XVIII a publicar el artículo en las actas de la conferencia en Acceso Abierto (Open Access) en diversos formatos digitales (PDF, HTML, EPUB) e integrarlos en diversas plataformas online como repositorios y bases de datos bajo la licencia CC:

Attribution-NonCommercial-ShareAlike 4.0 International (CC BY-NC-SA 4.0) https://creativecommons. org/licenses/by-nc-sa/4.0/.

Ni APANAC XVIII ni los editores son responsables del contenido ni de las implicaciones de lo expresado en el artículo. 